\title{
Bandwidth and Routing Optimization in Wireless Cellular Networks with Relays
}

\author{
Jingping Ji and Wei Yu \\ Department of Electrical and Computer Engineering \\ University of Toronto, Toronto, Canada M5S 3G4 \\ Emails: jp.ji@utoronto.ca,weiyu@comm.utoronto.ca
}

\begin{abstract}
This paper aims to quantify the performance improvement due to the use of fixed relays in the uplink of a wireless cellular network. Consider an orthogonal frequency division multiplex (OFDM) based cellular network in which each cell consists of a base station, multiple mobile users, and a number of relays. The questions of which frequency tones each link should use, whether the mobile station should communicate directly to the base station or though a relay, and how much power should be allocated on each frequency tone, form a simultaneous routing, frequency planning, and power allocation problem. This paper presents a dual decomposition approach to this problem and illustrates that while the use of relays does not necessarily increase the total cell throughput, it significantly improves the minimum common rate achievable across all the mobile users. Thus, the main benefit for deploying relays is in the improvement in fairness, rather than the total throughput.
\end{abstract}

\section{INTRODUCTION}

The use of relays to improve the performance of wireless cellular systems has been a subject of intense research activities in recent years. Relays have been shown to be capable of improving the capacity, coverage, and reliability of wireless connections in various settings (e.g. [1]-[7]). However, the deployment of relays in a cellular network also introduces significant practical challenges. Not only does the use of relays increase deployment cost, the network planning and resource allocation problem in relay networks also becomes more complicated.

This paper aims to quantify the performance improvement due to the use of relays from a system point of view by considering a resource allocation problem in the uplink of a wireless cellular network with fixed relay infrastructure. We consider an orthogonal frequency division multiple access (OFDMA) based system and dynamically optimize which frequency tones each mobile station (MS) and relay station (RS) should use, whether the MS should communicate directly to the base station (BS) or though a relay, and how much power should be used on each frequency tone. We use a dual decomposition technique and show that such a simultaneous routing, frequency planning, and power allocation problem can be solved efficiently. Further, by comparing with traditional networks while taking deployment cost and fairness across the users into account, we illustrate that the main benefit of relay deployment is in improving the minimum supportable data rate rather than the overall throughput.

Resource allocation issues for wireless cellular networks with relays have been considered extensively in the literature.
On a link-to-link level, various optimization techniques for the allocation of transmit power and frequency in relay networks have been investigated in [8]-[11]. On a network level, load balancing, quality-of-service and proportional fairness considerations have been given in [12], [13] and [14], respectively. A central technique emerging from many of these network optimization studies is the idea of dual decomposition. In this realm, [8] studied the joint optimization of relay strategies and resource allocation, while [15] and [16] employed a cross-layer approach and decomposed the problem into a weighted rate sum maximization problem in the physical layer and a routing problem in the network layer.

This paper also advocates the use of the dual decomposition approach, but offers a simplified formulation as compared to [15], [16] in the following sense. In a cellular network with fixed relays, the mobile users are typically either much closer to the BS, or much closer to one of the relays. Thus, the diversity combining gain from the multiple paths (either from MS directly to the BS or from MS to BS through multiple RSs) is likely to be marginal. Instead of including diversity gain as in [15], [16], this paper uses a multicommodity flow model and considers the relays as performing simple multihop decode-and-forward. In this case, the network optimization and resource allocation problem becomes that of deciding whether to transmit directly to the BS or through an RS, and if transmitting via an RS, which RS should be chosen. Further, such a question must be jointly answered with that of power and frequency allocation.

The problem formulation used in this paper is closely related to that of dynamic spectrum management and frequency planning for traditional OFDM networks (e.g. [17]). In particular, we utilize a theoretical result [18], [19], which states that the dual decomposition technique, when applied to OFDM systems with a large number of tones, results in zero duality gap, even when the original optimization problem is nonconvex. This observation leads to efficient spectrum management algorithms and allows a low-complexity solution for the frequency planning problem in OFDM systems [20].

A key consideration in resource allocation problems for cellular networks is the management of intracell and intercell interference. Current generation of cellular networks are designed so that only one mobile is active at any given frequency/time, thus completely eliminating intracell interference. This paper follows a similar approach whereas only a 


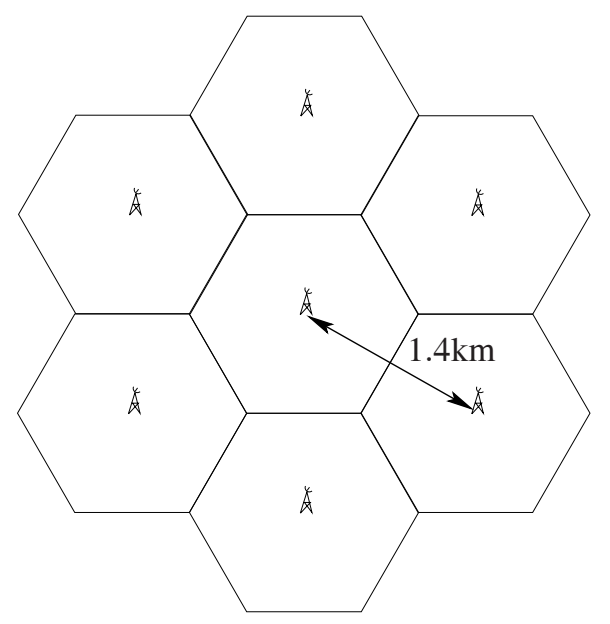

Fig. 1. Baseline scenario with 7 cells and $1.4 \mathrm{~km}$ base-station to base-station distance

single link within each cell (either MS-RS, MS-BS or BS-RS) is allowed to be active at any given frequency/time. However, instead of studying each cell in isolation, this paper takes intercell interference into account by following an iterative per-cell optimization approach. We consider a scenario where multiple cells each optimize routing, power allocation and frequency planning within its own cell while treating outof-cell interference as noise-thus achieving an interference avoidance effect for the overall network.

\section{Problem Setup}

As the baseline scenario, this paper considers a traditional hexagonally tiled cellular network as shown in Fig. 1. The mobile users are randomly distributed, each communicating with its own base-station directly, but on non-overlapping frequency tones within each cell.

To compare the baseline system with a relay network in a fair manner, one must explicitly recognize the additional cost associated with deploying the fixed relay infrastructure. Toward this end, this paper assumes that the cost of a RS is a fraction (e.g. 1/3) of the cost of a BS. Thus, by doubling the cell area (thereby reducing the number of BS's required to cover a geographical area by half), one may deploy several (e.g. 3) relays within each cell, while keeping the deployment cost the same as the baseline scenario.

Fig. 2 illustrates such a physical setup, where three relays are deployed in a ring centered around the BS, placed at 120 degrees apart and at a distance $\frac{2}{3}$ of the cell radius. In this particular case, the cell diameter in the baseline system is chosen to be $1.4 \mathrm{~km}$. In the relay-assisted network, the cell diameter is chosen to be $1.98 \mathrm{~km}$, reflecting the doubling of the cell area. In both cases, the mobile users are randomly distributed with the same area density.

In the baseline scenario, optimal frequency and power allocation problem is essentially that of optimally assigning users to each frequency tone. In this case, a dual decomposition approach [20] can be used to find the global optimum efficiently. This approach together with the iterative per-cell

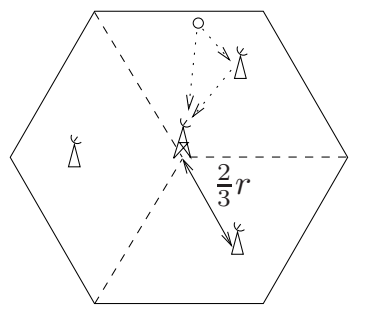

- Mobile Station

$\longleftarrow$ Relay Station

‡ Base Station

Fig. 2. Relay scenario: 3 relays at $\frac{2}{3} r, 1.98 \mathrm{~km}$ cell diameter

optimization, which takes into account intercell interference, is adopted in this paper to produce the baseline performance.

The relay-assisted scenario is considerably more complicated. In this case, mobile users have a choice of either communicating directly to the base-station or routing through a relay. Further, such a choice is coupled with frequency assignment and power allocation. In the next section, we provide an optimization framework for solving such a problem in an efficient manner.

\section{Optimization fOR A Relay Network}

\section{A. Illustrative Example}

Consider the illustrative example shown in Fig. 3 with two MS's, two RS's and one BS, labeled as $\mathrm{U}_{1}, \mathrm{U}_{2}, \mathrm{R}_{\mathrm{A}}, \mathrm{R}_{\mathrm{B}}$, and $\mathrm{B}_{0}$, respectively. There are many ways with which the relays may help the communication between the BS and the MS's. For example, the relay may decode then forward a re-encoded copy of the received signal, or the relay may simply amplify the received signal. Further, the receiver (either BS or MS) may use diversity combining technique to combine signals from multiple paths. As mentioned earlier, this paper focuses on decode-and-forward only, and ignores the possibility of diversity combining. In this case, the main design parameter becomes that of routing.

Consider the uplink scenario in Fig. 3. The MS $U_{1}$ may choose to communicate directly with the $\mathrm{BS}$ via $h_{10}$, or via relay A through $h_{1 \mathrm{~A}}-h_{\mathrm{A} 0}$, or via relay $\mathrm{B}$ through $h_{1 \mathrm{~B}}-h_{\mathrm{B} 0}$. Which link should be used depends largely on the relative strengths of these channels. Alternatively, the MS may choose to transmit part of its data directly to the BS, while transmitting part of its data via relay A and part via relay B. This can be the best thing to do when the channels are frequency selective, in which case one channel may be stronger than another in some frequency tones, but weaker in some other frequency tones. Similarly, $\mathrm{U}_{2}$ may partially transmit its data directly to the $\mathrm{BS}$ and partially via relay $\mathrm{B}$. In this case, $\mathrm{U}_{2}$ and $\mathrm{U}_{1}$ in fact compete for resources at relay B, as relay B must ensure that it can support the sum of rates from the two mobile users. Network constraints such as this are flow conservation constraints. They can be captured in a multicommodity flow model as shown in the following.

Consider the uplink scenario. Label the collection of all nodes (including MS's, RS's and the BS) as $k=1, \ldots, K$ and the collection of communication links as $l=1, \ldots, L$. The network topology can be represented by a node-link incidence 


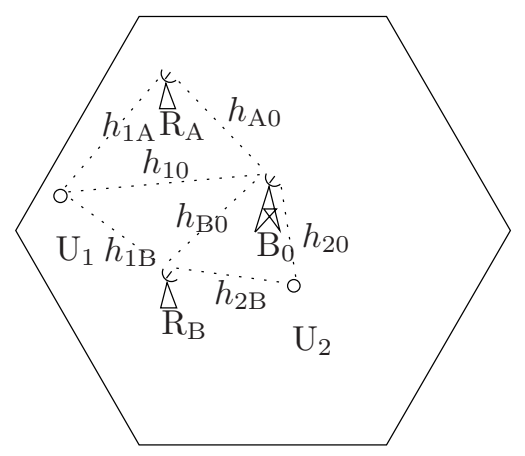

Fig. 3. A wireless cell with two users and two relays

matrix $A \in \mathfrak{R}^{K \times L}$ with the elements $a_{k l}$ defined as follows:

$$
a_{k l}=\left\{\begin{array}{cl}
1 & \text { if } k \text { is the start node for link } l \\
-1 & \text { if } k \text { is the end node for link } l \\
0 & \text { else }
\end{array}\right.
$$

Let $\boldsymbol{r}=\left[r_{1}, \cdots, r_{L}\right]^{T}$ be a vector of $L$ link rates, and $s=\left[s_{1}, \cdots, s_{K}\right]^{T}$ be a vector of $K$ source rates. The flow conservation constraint can be expressed as (see e.g. [21], [22])

$$
A \boldsymbol{r} \geq s
$$

For uplink transmission in a network with dedicated relays, the source rates at the BS and RS are 0, while the source rates at the MS's are the minimum supportable user rates.

\section{B. Optimization Formulation}

This paper formulates an optimization problem of maximizing the user rates across all the MS's, while maintaining a minimal common supportable rate for all users to ensure fairness. The minimal common supportable rate is incorporated in the vector $s$. The user rate $R_{k}$ for the $k$ th node is the sum of rates of all links originating from node $k$, expressed as

$$
R_{k}=\sum_{l \in \boldsymbol{O}(k)} r_{l}
$$

where $\boldsymbol{O}(k)$ denotes the set of links originating from node $k$.

In an OFDM system, the link rate is the sum of bit rates across the $N$ frequency tones, i.e.,

$$
r_{l}=\sum_{n=1}^{N} b_{l}(n)
$$

where $b_{l}(n)$ is the bit rate of link $l$ on tone $n$. The bit rate is related to power allocation as follows:

$$
b_{l}(n)=\log \left(1+\frac{p_{l}(n) h_{l}^{2}(n)}{\sigma_{l}^{2}(n)}\right)
$$

where $p_{l}(n)$ is the power allocated to link $l$ on tone $n, h_{l}(n)$ is the channel gain for link $l$ on tone $n$, and $\sigma_{l}^{2}(n)$ is the combined out-of-cell interference and noise on link $l$ at tone $n$. The network is subject to a power constraint at each node with the power usage at node $k$ expressed as

$$
P_{k}=\sum_{l \in \boldsymbol{O}(k)} \sum_{n=1}^{N} p_{l}(n)
$$

We can now write down the per-cell joint routing, power and bandwidth allocation problem as follows. Let $S$ denote the set of all links originating from mobile users (but not relays). The objective is to maximize the sum of mobile rates, subject to a minimal common supportable rate across all the mobiles:

$$
\begin{array}{cl}
\max _{\left\{p_{l}(n)\right\}} & \sum_{l \in \mathbf{S}} \sum_{n=1}^{N} b_{l}(n) \\
\text { s.t. } \quad & A \boldsymbol{r} \geq \boldsymbol{s} \\
& \sum_{l \in \boldsymbol{O}(k)} \sum_{n=1}^{N} p_{l}(n) \leq P_{k}^{\max }, \quad k=1, \cdots, K \\
& p_{l}(n) \geq 0, \quad l=1, \cdots, L, \quad n=1, \cdots, N \\
& p_{l}(n) p_{j}(n)=0 \quad \forall l \neq j
\end{array}
$$

The optimization variables are the power allocations $p_{l}(n)$. The constraints are the routing constraint, the maximum pernode power constraint $P_{k}^{\max }$, and the constraint that no two links share the same frequency within each cell. This last constraint stems from the assumption that no frequency reuse is allowed within each cell.

\section{Solution via Dual Decomposition}

The optimization problem formulated in (7) is a nonconvex problem because of the frequency nonoverlapping constraint $p_{l}(n) p_{j}(n)=0, \forall l \neq j$. Fortunately, nonconvex constraint of this type can be handled efficiently for the OFDM optimization problem via dual decomposition due to the following result in [18]-[20]. In the limit of large number of OFDM tones, this particular nonconvex problem has zero duality gap. Further, the evaluation of the dual objective has polynomial complexity, resulting in efficient algorithms for identifying the global optimum solution of (7).

We begin the treatment by writing down the Lagrangian:

$$
\begin{aligned}
L\left(p_{l}(n), \boldsymbol{\lambda}, \boldsymbol{\omega}\right) & =\sum_{l \in \mathbf{S}} \sum_{n=1}^{N} b_{l}(n)+\boldsymbol{\lambda}^{T}(A \boldsymbol{r}-\boldsymbol{s}) \\
& +\sum_{k=1}^{K} \omega_{k}\left(P_{k}^{\max }-\sum_{l \in \boldsymbol{O}(k)} \sum_{n=1}^{N} p_{l}(n)\right)
\end{aligned}
$$

where the dual variables are $\boldsymbol{\lambda} \in \mathfrak{R}^{K \times 1}$ and $\boldsymbol{\omega} \in \mathfrak{R}^{K \times 1}$. It is possible to rewrite this expression so that it is decoupled across the $N$ frequency tones as seen below:

$$
\begin{aligned}
L\left(p_{l}(n), \boldsymbol{\lambda}, \boldsymbol{\omega}\right) & =\sum_{k=1}^{K} \omega_{k} P_{k}^{\max }-\boldsymbol{\lambda}^{T} \boldsymbol{s} \\
+ & \sum_{n=1}^{N} \sum_{l=1}^{L}\left(\left(\delta_{l}+\boldsymbol{\lambda}^{T} A_{l}\right) b_{l}(n)-\omega_{l} p_{l}(n)\right)
\end{aligned}
$$

where $A_{l}$ is the $l$ th column of the matrix $A, \omega_{l}$ is defined such that $\omega_{l}=\omega_{k}$ for all links originating from node $k$, and $\delta_{l}$ is defined such that $\delta_{l}=1$ for all links originating from sources (i.e. $l \in \mathbf{S}$ ) and $\delta_{l}=0$ otherwise. 
The dual objective $g(\boldsymbol{\lambda}, \boldsymbol{\omega})$ can now be written down as

$$
\begin{aligned}
g(\boldsymbol{\lambda}, \boldsymbol{\omega})= & \max _{\left\{p_{l}(n)\right\}} L\left(p_{l}(n), \boldsymbol{\lambda}, \boldsymbol{\omega}\right) \\
\text { s.t. } \quad p_{l}(n) \geq 0, \quad l=1, \cdots, L & \quad p_{l}(n) p_{j}(n)=0 \quad \forall l \neq j
\end{aligned}
$$

The above maximization problem can be decomposed in a tone-by-tone basis. Define

$$
q_{n}\left(p_{l}(n), \boldsymbol{\lambda}, \boldsymbol{\omega}\right)=\sum_{l=1}^{L}\left(\left(\delta_{l}+\boldsymbol{\lambda}^{T} A_{l}\right) b_{l}(n)-\omega_{l} p_{l}(n)\right) .
$$

The maximization in (10) then becomes

$$
g(\boldsymbol{\lambda}, \boldsymbol{\omega})=\sum_{k=1}^{K} \omega_{k} P_{k}^{\max }-\boldsymbol{\lambda}^{T} \boldsymbol{s}+\sum_{n=1}^{N} \max _{\left\{p_{l}(n)\right\}} q_{n}\left(p_{l}(n), \boldsymbol{\lambda}, \boldsymbol{\omega}\right)
$$

subject to the same constraints as in (10).

We can solve the maximization problem in (12) explicitly with the following line of reasoning. Since $q_{n}\left(p_{l}(n), \boldsymbol{\lambda}, \boldsymbol{\omega}\right)$ is composed of $L$ terms each depending only on the power allocation on the $l$ th link $p_{l}(n)$, and the constraint $p_{l}(n) p_{j}(n)=0$ $\forall l \neq j$ enforces that only one link may be active at any given frequency tone, the maximization of $q_{n}\left(p_{l}(n), \boldsymbol{\lambda}, \boldsymbol{\omega}\right)$ is equivalent to the maximization over $L$ terms, i.e.

$$
\begin{aligned}
\max _{\left\{p_{l}(n)\right\}} q_{n}\left(p_{l}(n), \boldsymbol{\lambda}, \boldsymbol{\omega}\right) \\
=\max _{\left\{p_{l}(n)\right\}} \sum_{l=1}^{L}\left(\left(\delta_{l}+\boldsymbol{\lambda}^{T} A_{l}\right) b_{l}(n)-\omega_{l} p_{l}(n)\right) \\
=\max _{l} \max _{\left\{P_{l}(n)\right\}}\left(\left(\delta_{l}+\boldsymbol{\lambda}^{T} A_{l}\right) b_{l}(n)-\omega_{l} p_{l}(n)\right)
\end{aligned}
$$

subject to the constraint $p_{l}(n) \geq 0$. The above maximization problem can be easily solved as $b_{l}(n)$ is a concave function of $p_{l}(n)$. Hence the dual objective function, which now becomes

$$
\begin{aligned}
& g(\boldsymbol{\lambda}, \boldsymbol{\omega})=\sum_{k=1}^{K} \omega_{k} P_{k}^{\max }-\boldsymbol{\lambda}^{T} s \\
& \quad+\sum_{n=1}^{N} \max _{l} \max _{\left\{p_{l}(n)\right\}}\left(\left(\delta_{l}+\boldsymbol{\lambda}^{T} A_{l}\right) b_{l}(n)-\omega_{l} p_{l}(n)\right),
\end{aligned}
$$

can be evaluated efficiently in polynomial time.

To obtain the solution to the original optimization problem, it remains to identify the optimal dual variables, which can be found by solving the dual problem

$$
\begin{array}{ll}
\min _{\boldsymbol{\lambda}, \boldsymbol{\omega}} & g(\boldsymbol{\lambda}, \boldsymbol{\omega}) \\
\text { s.t. } & \boldsymbol{\lambda} \geq 0 \\
& \boldsymbol{\omega} \geq 0
\end{array}
$$

The optimal dual variables $\lambda^{*}$ and $\boldsymbol{\omega}^{*}$ can be found via a dual update method, such as the ellipsoid method. The final step is to recover the optimal primal variables by allowing timesharing and by solving a linear programming problem [23].

\section{PERformance Evaluation}

\section{A. Simulation Setup}

The objective of this paper is to evaluate the benefit of relaying in a cellular network. Toward this end, we simulate the uplink of a baseline network with 7 hexagonal neighboring cells with 9 users per cell and with a cell diameter of $1.4 \mathrm{~km}$. We then simulate a relay network in which the cell area is doubled (with diameter $1.98 \mathrm{~km}$ ), but with 3,4 or 5 relays deployed within each cell. As mentioned earlier, the rationale for doubling the cell size is to account for the increased deployment cost associated with the relays. In all scenarios, the relays are positioned in a ring around the base station at a distance of $\frac{2}{3} r$, where the cell radius $r=0.99 \mathrm{~km}$. The angles between the relays are 120 degrees for the case of 3 relays, 90 degrees for the case with 4 relays, and 72 degrees for the case with 5 relays. Hence, each relay implicitly covers a pieshaped sector within each cell. We place 6 users per sector for the 3-relay case, 5 users per sector for the 4-relay case, and 4 users per relay sector for the 5-relay case, keeping the mobile density roughly equal. Finally, we also simulate a case of 3 relays and 6 mobile users per sector, but with relays located $\frac{1}{3} r$ away from the base station. This last scenario explores the effect of relay location within a cell.

The simulation parameters are modeled after the Mobile WiMAX standard [24] with the number of OFDM tones $N=$ 1024. For simplicity, we do not take into consideration the pilot sub-carriers, null sub-carriers, nor do we follow the subchannelization described in [24]. The propagation model used in this simulation incorporates a multi-path time delay profile with Rayleigh fast fading, shadowing, and the path loss effect. The multi-path time delay profile is taken from the ITU-R M.1225 Pedestrian A model [25]. The shadowing effect is modeled using a Gaussian random variable with zero mean and a standard deviation of $8 \mathrm{~dB}$.

The simulation follows the optimization framework outlined in Section III on a per-cell basis. We maximize the sum of mobile rates, while maintaining a minimum common rate. Intercell interference is accounted for through an iterative per-cell optimization process over the 7 cells, with intercell interference updated at the end of the each iteration.

A pertinent performance metric of interest is the achievable minimum common rate for all mobile users in the entire network. The minimum common rate is set in the constraint $A r \geq s$ in the optimization problem. For each simulation scenario, the largest minimum supportable common rate is found via a bisection search over $s$. We then report the minimum common rate and the maximum sum rate under this common rate for each scenario.

\section{B. Results}

Table I presents the performance results for the various simulation scenarios. Note that the minimum common rate and the sum of source rate are the averaged values over a number of independent simulations. Comparing the performance of the baseline scenario (BL) with various relay scenarios, it is clear 
TABLE I

PERFormance COMPARISON OF SimUlation SCEnARIOS

\begin{tabular}{|l|c|c|c|c|c|}
\hline Scenario & BL & RS 1 & RS 2 & RS 3 & RS 4 \\
\hline Cells Simulated & 7 & 7 & 7 & 7 & 7 \\
\hline \# Sim. Avged & 5 & 6 & 2 & 2 & 2 \\
\hline Relays Per Cell & 0 & 3 & 4 & 5 & 3 \\
\hline Relay Distance & N/A & $\frac{2}{3} r$ & $\frac{2}{3} r$ & $\frac{2}{3} r$ & $\frac{1}{3} r$ \\
\hline MU Per Cell & 9 & 18 & 20 & 20 & 18 \\
\hline Cell Diameter $(\mathrm{km})$ & 1.4 & 1.98 & 1.98 & 1.98 & 1.98 \\
\hline Cell Area $\left(\mathrm{km}^{2}\right)$ & 1.54 & 3.08 & 3.08 & 3.08 & 3.08 \\
\hline Min C Rate $(\mathrm{Mbps})$ & 0.193 & 0.583 & 0.972 & 0.705 & 0.578 \\
\hline Sum S Rate $(\mathrm{Mpbs})$ & 96.4 & 75.4 & 80.2 & 72.7 & 87.2 \\
\hline
\end{tabular}

that the relays provide a significant benefit to the minimum common rate, while actually somewhat lowering the overall sum rate. The maximum minimum common rate triples with the deployment of 3 relays (583kbps vs $193 \mathrm{kbps}$ ). Thus, the major benefit of fixed relay infrastructure is to make the system more equitable, rather than increasing the overall throughput.

This is quite a desirable result from an operator's point of view, as fairness is often more important from a revenue perspective as compared to the overall throughput. This result is also intuitively sensible, as the cell edge users are fundamentally limited by path loss and intercell interference. The deployment of relays significantly improves the distancedependent path loss, as the mobiles at the cell edge are much closer to the relays than to the BS. The relays also offer the mobile users the choice of communicating directly to the BS or routing through the nearest relay, thus achieving a path diversity. Finally, deploying relays does not appear to affect the intercell interference significantly in this uplink scenario.

The observation that the network with relays does not improve the overall throughput may be related to the fact that frequency reuse is not allowed within each cell in the current optimization setup. Any information that is routed through a relay uses up additional bandwidth resources and may potentially decrease the area spectral efficiency in the system. As the system with relays covers an area twice that of the baseline scenario, future work where frequency reuse is judiciously allowed within each cell has the potential to further improve the performance of relay networks.

Finally, we note that increasing the number of relays seems to give further gains in the minimum common rate. Also, changing position of the relay within the cell from $\frac{2}{3} r$ to $\frac{1}{3} r$ does not seem to have a significant effect on performance.

\section{CONCLUSION}

This paper makes progress in the optimization of OFDM networks with relays. We find a solution to the simultaneous routing, frequency planning, and power allocation problem in a cellular network with fixed relay infrastructure and conclude that the major benefit of relays is to make the system more equitable while extending coverage.

\section{REFERENCES}

[1] A. Sendonaris, E. Erkip, and B. Aazhang, "User cooperative diversity. Part I: System description. Part II: Implementation aspects and perfor- mance analysis.," IEEE Trans. Commun., vol. 51, no. 11, pp. 1927 1948, Nov 2003.

[2] N. Esseling, B. H. Walke, and R. Pabst, "Performance evaluation of a fixed relay concept for next generation wireless systems," in Proc. IEEE Int. Symp. PIMRC, Sep 2004.

[3] T. Irnich, D.C. Schultz, R. Pabst, and P. Winert, "Capacity of a relaying infrastructure for broadband radio coverage of urban areas," in IEEE Veh. Tech. Conf., Fall 2003, Orlando, USA.

[4] D. Schultz, B. Walke, R. Pabst, and T. Irnich, "Fixed and planned relay based radio network deployment concepts," in 10th Wireless World Research Forum, Oct 2003, New York, USA.

[5] H. Hu, H. Yanikomeroglu, D. Falconer, and S. Periyalwar, "Range extension without capacity penalty in cellular networks with digital fixed relays," in Proc. IEEE Globecom, Dec 2004, pp. 3053-3057.

[6] J. Cho and Z. Haas, "On the throughput enhancement of the downstream channel in cellular radio networks through multihop relaying," IEEE J. Sel. Areas Commun., vol. 22, no. 7, pp. 1206-1219, Sep 2004.

[7] A. So and B. Liang, "Enhancing WLAN capacity by strategic placement of tetherless relay points," IEEE Trans. Mobile Computing, vol. 6, no. 5, pp. 1536-1233, May 2007.

[8] T. Ng and W. Yu, "Joint optimization of relay strategies and resource allocations in a cooperative cellular network," IEEE J. Sel. Areas Commun., vol. 25, no. 2, pp. 328-339, February 2007.

[9] G. Li and H. Liu, "Resource allocation for OFDMA relay networks with fairness constraints," IEEE J. Sel. Areas Commun., vol. 24, no. 11, pp. 2061-2069, Nov 2006.

[10] J. Wang, Y. Zhao, and T. Korhonen, "Cross layer optimization with complete fairness constraints in OFDMA relay networks," in Proc. IEEE Globecom, Dec 2008.

[11] R. Kwak and J. M. Cioffi, "The subchannel-allocation for OFDMA relaying downlink systems with total power constraint," in Proc. IEEE Globecom, Dec 2008.

[12] Y. Liu, R. Hoshyar, X. Yang, and R. Tafazolli, "Integrated radio resource allocation for multihop cellular networks with fixed relay stations," IEEE J. Sel. Areas Commun., vol. 24, no. 11, pp. 2137-2146, Nov 2006.

[13] J. Tang and X. Zhang, "Cross-layer resource allocation over wireless relay networks for quality of service provisioning," IEEE J. Sel. Areas Commun., vol. 25, no. 4, pp. 645-655, May 2007.

[14] W.-G. Ahn and H.-M. Kim, "Proportional fair scheduling in relay enhanced cellular ofdma systems," in Proc. IEEE Int. Symp. PIMRC, Sep 2008.

[15] L. You, M. Song, and J. Song, "Cross-layer optimization for fairness in OFDMA cellular networks with fixed relays," in Proc. IEEE Globecom, Dec 2008.

[16] S.-J. Kim, X. Wang, and M. Madihian, "Optimal resource allocation in multi-hop OFDMA wireless networks with cooperative relay," IEEE Trans. Wireless Commun., vol. 7, no. 5, pp. 1833-1838, May 2008.

[17] C.Y. Wong, R.S. Cheng, K.B. Letaief, and R.D. Murch, "Multiuser OFDM with adaptive subcarrier, bit, and power allocation," IEEE J. Sel. Areas Commun., vol. 17, pp. 1747- 1758, Oct 1999.

[18] W. Yu and R. Lui, "Dual methods for nonconvex spectrum optimization of multicarrier systems," IEEE Trans. Commun., vol. 54, no. 7, pp. 1310-1322, Jul 2006

[19] Z. Luo and S. Zhang, "Dynamic spectrum management: Complexity and duality," IEEE J. Sel. Top. Signal Processing, vol. 2, no. 1, pp. 57-73, Feb 2008.

[20] W. Yu, R. Lui, and R. Cendrillon, "Dual optimization methods for multiuser orthogonal frequency division multiplex systems," in Proc. IEEE Globecom, Nov 2004, pp. 225- 229.

[21] L. Xiao, M. Johansson, and S. Boyd, "Simultaneous routing and resource allocation via dual decomposition," IEEE Trans. Commun., vol. 52, pp. 1136- 1144, Jul 2004.

[22] J. Yuan and W. Yu, "Joint source coding, routing and power allocation in wireless sensor networks," IEEE Trans. Commun., vol. 56, no. 6, pp. 886-898, Jan 2008.

[23] Z. Luo and S. Zhang, "Duality gap estimation and polynomial time approximation for optimal spectrum management," To appear in IEEE Trans. Signal Processing., 2009.

[24] WiMAX Forum, "Mobile WiMAX Part I: A Technical Overview and Performance Evaluation," [Online], Available: http://www.wimaxforum.org/technology/downloads/.

[25] ITU-R, "M.1225 Guidelines for evaluation of radio transmission technologies for IMT-2000," [Online], Available: http://www.itu.int/rec/RREC-M/e. 